\title{
Labyrinthe
}

$27 \mid 2007$ (2)

La fin des disciplines?

\section{Si parler va sans dire. Du logos et d'autres ressources, de François Jullien}

\section{Bérengère Hurand}

\section{OpenEdition}

1 Journals

\section{Édition électronique}

URL : https://journals.openedition.org/labyrinthe/1976

DOI : $10.4000 /$ labyrinthe. 1976

ISSN : 1950-6031

\section{Éditeur}

Hermann

\section{Édition imprimée}

Date de publication : 4 juillet 2007

Pagination : 119-124

ISBN : 978-2-9526131-4-9

\section{Référence électronique}

Bérengère Hurand, «Si parler va sans dire. Du logos et d'autres ressources, de François Jullien »,

Labyrinthe [En ligne], 27 | 2007 (2), mis en ligne le 25 mars 2011, consulté le 26 avril 2022. URL : http:// journals.openedition.org/labyrinthe/1976 ; DOI : https://doi.org/10.4000/labyrinthe.1976 


\section{SI PARLER VA SANS DIRE Du logos et d' autres ressources de François Jullien*}

Bérengère HURAND berhurand@yahoo.fr

L'avant-dernier ouvrage du sinologue François Jullien porte un regard passionnant sur le logos aristotélicien à partir duquel s'est construit l'empire conceptuel d'Occident ${ }^{1}$. Confrontée ici à son autre (ignoré) d'Orient, la parole grecque laisse deviner la faiblesse et l'artifice de la notion de vérité - ou plutôt du dire vrai - qu'on pouvait penser universelle et absolue, dès lors qu'elle avait été posée par les fils de Socrate comme la seule voie possible pour s'élever au-dessus de la grouillante diversité du réel, où l'on se perd. Car dire, au sens grec, c'est toujours dire quelque chose, définir, circonscrire l'être et supposer la puissance de la parole à atteindre l'identité de la chose. Dire, c'est aussi éviter de se contredire, respecter le dire vrai antérieur ou postérieur, la vérité axiomatique ou la conformité avec une réalité classée, catégorisée, abstraite. À l'autre bout du monde, la Chine taoïste a commencé à penser «non pas selon le pli de l'Être et donc de l'entité-identité, mais selon la capacité investie et se déployant sans cesse dans le grand procès du monde» (p. 38), proche en cela de la sagesse héraclitéenne qui indique, pointe vers, mais ne dit pas; ou, plus précisément, qui dit sans scinder. Il $\mathrm{y}$ a là matière à comparer.

Suivre le tao, la voie ou le procès, ce n'est pas imiter l'être: car la voie imite son propre jaillissement. François Jullien nous mène sur les traces de l'allusif, de l'oblique, de la transformation; il aborde un monde où parler - et donc penser - par identité, opposition et contraires

* Paris, Le Seuil, coll. «L'ordre philosophique», 2006, 192 p., $18 €$.

1. Si parler va sans dire, consacré au logos, est le premier volume d'une trilogie. Les deux suivants traiteront des notions grecques d'eidos et de theos selon le même principe de mise à l'épreuve de l'altérité conceptuelle. 
n'est pas de mise. Au lieu de s'ébattre raisonnablement dans le champ du logos borné par le principe et la fin, il s'agit de faire «déborder la parole de la signification» (p. 51), de rejoindre la «processivité diffuse, invisible, infinie» du tao, vase vide " par où du réel intarissablement se déploie» (p. 61). Il faut s'accrocher à l'émergence permanente des choses pour ne pas se figer, s'enliser dans le marais de leur définition. Ainsi, attribuer à quelqu'un une qualité morale, c'est en même temps soupçonner la personne qualifiée de pouvoir se soustraire à cette qualification: car il y a toujours «non-coïncidence entre effectivité et déterminité» (p. 78). Il n'y a rien de moins pieux qu'un bon chrétien, disait Bernanos; de même, pour le Laozi, «la vertu inférieure ne quitte pas la vertu, c'est pourquoi elle est sans vertu» (p. 78). La parole qualifiante laisse échapper la réalité de la chose; elle en suppose une compréhension factice. De cela en revanche on ne peut accuser la parole restée disponible, réservée, en retrait - la parole évolutive, celle de l'essor.

À s'en tenir là, on empêche certes la pensée de se scléroser; mais on évite aussi qu'un dialogue puisse naître dans l'antagonisme des thèses. Le refus taoïste d'un affrontement du logos contre lui-même sous l'autorité d'un principe de non-contradiction a rendu impossible la notion de vérité et, par conséquent, a pu compromettre l'émergence d'une contestation puis d'un affranchissement politique. Si le despotisme a des racines métaphysiques, c'est peut-être là qu'il faut les chercher: l'Empire chinois se serait fondé sur l'idée d'obéissance à ce qui vient de soi-même. François Jullien évoque la possibilité de cette généalogie; il y voit une filiation qui ne serait, somme toute, que naturelle. Mais là n'est pas l'avis de Jean-François Billeter, qui vient de publier Contre François Jullien $^{1}$. Pour ce dernier, le lien entre le taoïsme et le pouvoir impérial est plutôt à interpréter comme une adoption forcée: il soutient la thèse d'une appropriation délibérée des idées taoïstes par le premier empire chinois et la dynastie Han, auteurs d'un mythe dont les Jésuites auraient été les premières dupes et les premiers relais. L'œuvre entière de François Jullien témoignerait alors, de l'avis de Billeter, d'une vision naïve et idéologique de la civilisation chinoise. La pique est sévère pour le grand sinologue, soucieux de vulgariser le fruit de ses recherches - voir par exemple la Conférence sur l' efficacité ${ }^{2}$ où François Jullien donne des

\footnotetext{
1. Paris, Allia, 2006.
}

2. Paris, PUF, 2005. 
leçons de sinitude aux entrepreneurs français. Son erreur serait d'assimiler la philosophie chinoise tout entière à la métaphysique du tao, immanentiste, contemplative et résignée, démontrant par cet amalgame l'efficacité toujours aussi grande d'une propagande politique datant du $\mathrm{III}^{\mathrm{e}}$ siècle avant notre ère: la légitimité du pouvoir autoritaire est en effet assurée, si l'ordre des choses est conçu comme immanent et si la question des fins ne se pose pas à la conscience chinoise. Les idées de vérité, mais aussi de personne, de liberté, d'opposition et de contre-pouvoir ne peuvent pas y avoir de lieu. Or, confirmer comme le fait François Jullien les fondements culturels de ce verrouillage politique, c'est jouer le jeu du pouvoir répressif chinois. Plus qu'une erreur, c'est une véritable faute, une compromission inacceptable. Davantage encore: comprendre ainsi la philosophie chinoise accentue artificiellement, jusqu'à l'essentialiser, l'altérité de la culture chinoise. Cela justifie qu'elle reste extérieure à l'édifice culturel occidental, imperméable à la diffusion des idéaux démocratiques, rétive aux tentatives de dialogue interculturel. Cela réduit enfin la Chine au rôle de partenaire commercial bien pratique pour un Occident soucieux de conquérir des marchés: un partenaire dont il faut connaître, pour agir en conséquence, l'amoralité de la conception immanentiste du monde. Puisque la Chine obéit à des rapports de force spontanés, c'est-à-dire aux règles du marché, il devient inutile de lui imposer une législation économique découlant d'une conception transcendante de la justice'.

L'affaire ne manque pas d'intérêt; mais c'est aux sinologues qu'il revient de trancher, quant à la responsabilité historique de l'Empire chinois dans la diffusion du taoïsme. Le conflit ne porte heureusement pas sur l'interprétation de la pensée du tao; et rien n'empêche de tirer un profit tout égoïste de la lecture de François Jullien, en portant ce regard vertigineux et distancié qu'il suggère sur notre façon de parler et sur les exigences du dire. Si parler va sans dire, nous offrant une comparaison pré-

\footnotetext{
1. François Jullien vient de répondre à ces attaques dans Chemin faisant : connaître la Chine, relancer la philosophie, Paris, Seuil, coll. «L'ordre philosophique», 2007. Il ne se reconnaît pas coupable de construction de la culture chinoise; il revendique une réflexion à partir des concepts que ses lectures lui offrent, et qu'il travaille de manière an-historique dans un «auto-réfléchissement de l'humain ». S'il y a construction, ce n'est donc pas celle d'une histoire de la Chine et de sa pensée, mais une construction d'objets théoriques nés de la confrontation avec les concepts qu'a pu produire l'Occident. Voir également F. Jullien, «La Chine au miroir de l'Occident», Le Monde diplomatique, octobre 2006, p. 22-23; le texte est disponible à l'adresse : http://www.monde-diplomatique.fr/2006/10/JULLIEN/14010 [consulté le 25 mai 2007].
} 
cieuse entre Aristote et Zhuangzi, contemporains ( $\mathrm{V}^{\mathrm{e}}-\mathrm{IV}$ siècle av. J.-C.) qui s'ignorent, permet en effet d'adopter une position de Persan sur les conditions d'émergence de notre culture propre. Assimilable à première vue au relativisme sophiste, trop connu et déjà neutralisé par la réplique socratique, la métaphysique du tao représente pourtant tout autre chose: la possibilité pour la parole de se passer du logos, de ne pouvoir même l'envisager. Le dire taoïste est un dire au gré; il abandonne l'étale des choses, leur définition plate, pour imiter leur ainséité - le «de soi-même ainsi ». Plus qu'un simple relativisme, qu'une approche du devenir comparable à celle d'Héraclite, il s'agit d'une parole évolutive qui, tel le pivot, accueille ce vers quoi elle se tourne. Elle désocculte la parole en évitant de la figer dans l'assignation. Un des problèmes les plus aigus et récurrents de l'ontologie occidentale, la difficulté à toucher le réel par le moyen des mots, pourrait alors trouver une réponse dans la parole du tao qui réussit à dire, non pas le singulier défini par des catégories abstraites mais «l'infini de la prégnance», la fluidité, l'indistinction des choses.

Mais comment proposer un tel modèle à la pensée occidentale, s'il est impossible de l'y transposer? À lire les extraits que met à notre disposition Si parler va sans dire, la structure de nos langues occidentales se prête difficilement à la traduction du chinois, et la sagesse du tao, si elle n'est pas trahie par un discours reconstruit grammaticalement, se présente sous la forme hermétique d'une poésie saturée d'ellipses. Que tirer alors de ce qui se présente en dehors des repères familiers? Et quand même nous arriverions à pénétrer cette rhétorique, serions-nous prêts à abandonner, avec le logos, la vérité et la science? Car la parole du tao ne cherche pas la conformité avec le réel tel qu'il est: elle se déploie plutôt en conformation avec lui, tel qu'il advient. Elle s'accomplit dans l'encours, dans la transformation des choses. Elle se déprend d'elle-même et préfère la connivence à la connaissance. Comment la comparaison avec une sagesse aussi évasive, extérieure, floue, et finalement autre pourraitelle prétendre éclairer la pensée occidentale? La mise en parallèle des deux modes de pensée, selon le procédé classique, n'aurait donc pour résultat que d'accentuer leur différence fondamentale, afin de faire naître un soupçon relativiste sur nos préjugés ontologiques. Là semble être le seul but de François Jullien. Mais l'altérité radicale est dénoncée par Jean-François Billeter comme mode d'appréhension et de compréhension inadéquat, dangereux même - au niveau politique comme philosophique. D'où l'importance de prendre la mesure de cette altérité, en élar- 
gissant à sa postérité occidentale le terrain d'investigation, strictement aristotélicien, de François Jullien: car il est possible que le sentiment d'étrangeté naisse aussi de sa présentation quelque peu monolithique de deux systèmes plantés face à face.

La parole du tao se tient entre «il y a» et «il n'y a pas»; l'action, entre «j'agis» et «je n'agis pas». Le taoïste refuse de statuer, de se fixer sur une position sclérosante. Si elle se pose, la pensée n'arrive plus à suivre la réalité; elle court après quelque chose qui change: «l'infinie variation des choses» (p.124). Or, ce thème de la parole nécessairement en retard, toujours trop rigide, se développe aussi dans la philosophie occidentale dès qu'elle s'aperçoit de son impuissance à dire le singulier, le moi, le divers, le devenir - ou bien l'inassignable. Pour résoudre le problème, les fils d'Aristote ont exploré plusieurs pistes, dont la première, religieuse, voire obscurantiste, revendique sa folie et son extravagance. Elle renonce à signifier, à définir, à attribuer; elle établit son camp en un entre-deux-extrêmes qui les fait se rejoindre. On pense à la coïncidence des opposés, à la théologie négative par exemple. Or, la parole du tao que fait entendre François Jullien peut ressembler à cette rhétorique mystique. C'est une parole fade et sans saveur; mais c'est également une parole extravagante, évasive, folle, qui se retire du régime du sens, libre de toute intention, qui se répand et s'épanche - qui laisse se dire. Le deuxième hors-piste occidental est la voie poétique; là encore, le rapprochement est possible et bienvenu, selon l'auteur lui-même, avec une parole taoïste qui se prête davantage à la rêverie - voire à la méditation qu'à la construction intellectuelle. La troisième voie parallèle à la norme aristotélicienne est une signification qui cherche à dire sans atteindre, transgressant avec respect les principes d'identité et de non-contradiction. Il s'agit alors de circonscrire la chose, de l'approcher seulement en énonçant ce qui lui appartient, ce qui lui est attaché, ce qui est rapporté à elle. On use de métaphores, de synecdoques. N'avons-nous pas là une entreprise aussi intéressante que le dire au gré taoïste, et qui lui ressemble fort? Certes! à ceci près que la parole du tao renonce à placer la chose dans un lieu discursif. Elle vise l'entre-deux indicible, c'est-à-dire la processivité du réel. La parole occidentale en revanche, qu'elle soit poétique ou mystique, cherche toujours, si ce n'est à attraper la chose, du moins à attraper quelque chose de la chose - elle cherche toujours à fixer. Même le caractère incorrect, libre de l'assignation trouve ses limites dans la normalisation: les écarts sont répertoriés, classifiés, codi- 
fiés, et perdent ainsi la spontanéité de leur essor vers la chose. Ce que la parole du tao a su faire, comme François Jullien cherche à nous le montrer, c'est se déprendre de tout référentiel fixe; la pesanteur aristotélicienne nous rend sans doute impossible cette légèreté. 